\title{
Brushless DC Motor Drive During Speed Regulation With Adaptive Neuro-Fuzzy Inference System (ANFIS) Controller
}

\author{
Sakshi Solanki \\ (Department of Power System Engineering, Galgotias University, Greater Noida, India)
}

\begin{abstract}
This research paper demonstrates that Brushless DC (BLDC) motor for this stage is lavishly passed down by many industrial functions payable to the different features just like high speed range, high efficency and dynamic response. A prospective technology is used in this paper i.e. Adaptive Neuro-Fuzzy Inference System Controller(ANFIS) for controlling the speed of BLDC motor. In the previous research, ANN ( Artificial Neural Network) controller is used, which controlled the BLDC motor speed in an oppostion of PID controller. As BLDC is a non linear so for the most efficent results for controlling the speed, proposed ANFIS controller in which it require non mathematical model where a fuzzy file is generated therefore fuzzy speed controller used. It is a fuzzy system whose membership function parameters have been tuned using neuro adaptive learning methods similar to those used in training neural network. Here, the loading, generation, plotting and training of data is done. At the end it validated the trained FIS (Fuzzy Inference System) by using the software kit MATLAB ISIMULINK.
\end{abstract}

Keywords: Adaptive Neuro-Fuzzy Inference System (ANFIS) controller, ANFIS Editor, FIS model structure, Membership function parameters, Surface viewer.

\section{Introduction}

The BLDC motor is persistently resolved as a Permanent Magnet Synchronous Motor on a Trapezoidal Back EMF waveform shape. The Brushless DC motor is only one of motor which is promoting demands. The word "Brushless DC Motor" is generally identifies the sequence of machines of AC, position sensor of the rotor, solid-state inverter that conclusion in a drive system having characteristics of linear torque. Contrary to an article, BLDC motor are normally a type of permanent magnet synchronous motors. They got focused by DC voltage although the current commutation is executed by the solid state switches. The commutation swift are cohesive by the rotor position and the position of the rotor is concede either by position sensors or by sensor less techniques. This BLDC Motor encompass many assets by conventional DC motors same as long operating life, dynamic response is high, efficiency is high, speed vs. torque characteristics is amended, operation of noiseless, speed range is high, torque-weight ratio is also very high. The boost in the energy cost appetition higher entreaty of fluctuating speed PM motor drives. In addition, new agile generation of motor drives into the automobile industry, secure on the hybrid drives, progress a serious exigency for high efficient PM motor drives, and this was the ancestry of accent in the BLDC motors. The BLDC is an electronically controlled commutation system, fairly including a mechanical commutation, which is appropriate of brushed motors. Along with, the electromagnets do not move the rotation of the permanent magnets and the armature leftover static. The complication earned by this that how to bend the current to a moving armature. Admitting to do this, the brush system/commutator assembly is repaired by an intelligent electronic controller, which assassinates the same power distribution same as a Brushed DC motor. For the reason of the BLDC moves without brushes its lifetime used can be embossed and operation of maintenance also elude.

\subsection{ANFIS}

\section{Adaptive Neuro-Fuzzy Inference System (ANFIS) Controller}

Adaptive Neuro-Fuzzy Inference System (ANFIS) is a neuro fuzzy technique where the synthesis is built between the neural network and the fuzzy inference system. In the ANFIS, the criterion can be evaluated in alike a way that both the Sugeno and Tsukamoto fuzzy models are represented by the ANFIS architecture. The fuzzy logic proceeds into report the deception and confusion of the system that is real modeled while the neural network gives it a sight of flexibility. An adaptive neuro-fuzzy inference system (ANFIS) is a fuzzy system whose membership function parameters have been adapted using Neuro-Adaptive learning methods similar to those used in training neural networks. 


\subsection{ANFIS Controller}

In the modeling and observation control of any dynamical system, a controller is ought for the plant similarly it takes charge of all the disturbances and escorts back the system to its original state in a brace of seconds. Fuzzy logic is one of the most blooming applications in the control engineering field, which can be used to control different parameters of real-time systems. The logic emerged with neural networks output very compelling results. Now this merged technique of the attainment power of the NNs by the knowledge representation of Fuzzy Logic organized a new hybrid technique, called Neuro-Fuzzy networks . This technique presents a very good calculation of the speed and is vital to parameter variation. The ANFIS Controller does not require a mathematical model of the system and it works on a structure prepared from the knowledge base. In ANFIS Speed controller the inputs used are the speed error and rate of change of speed error. ANFIS generate fuzzy file therefore fuzzy logic controller is used as controller.

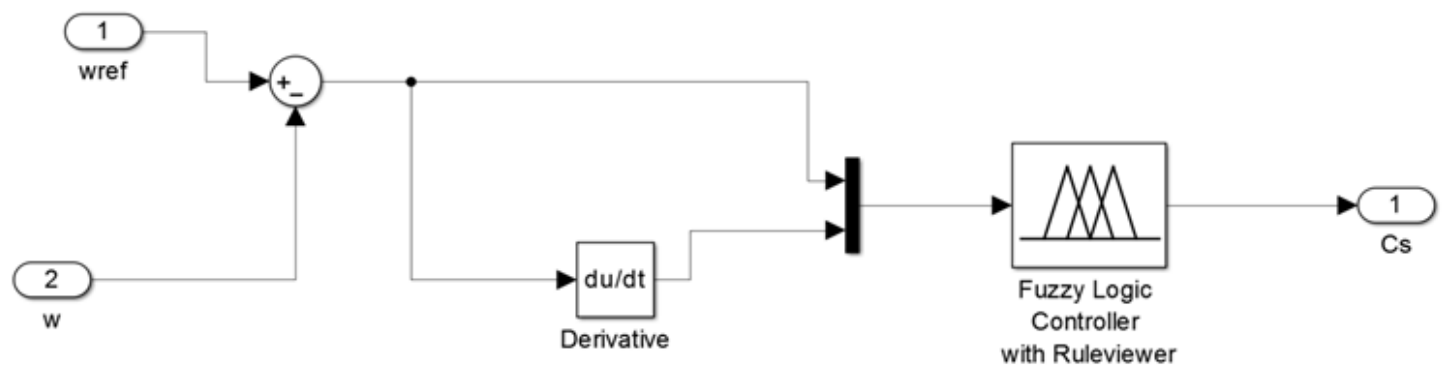

Figure1- ANFIS based BLDC controller

Fuzzy file generated using ANFIS editor

\section{ANFIS - Commands}

name: 'fisfile'

type: 'sugeno'

andMethod: 'prod'

orMethod: 'probor'

defuzzMethod: 'wtaver'

impMethod: 'prod'

aggMethod: 'sum'

input: [1x2 struct]

output: [1x1 struct]

rule: [1x25 struct]

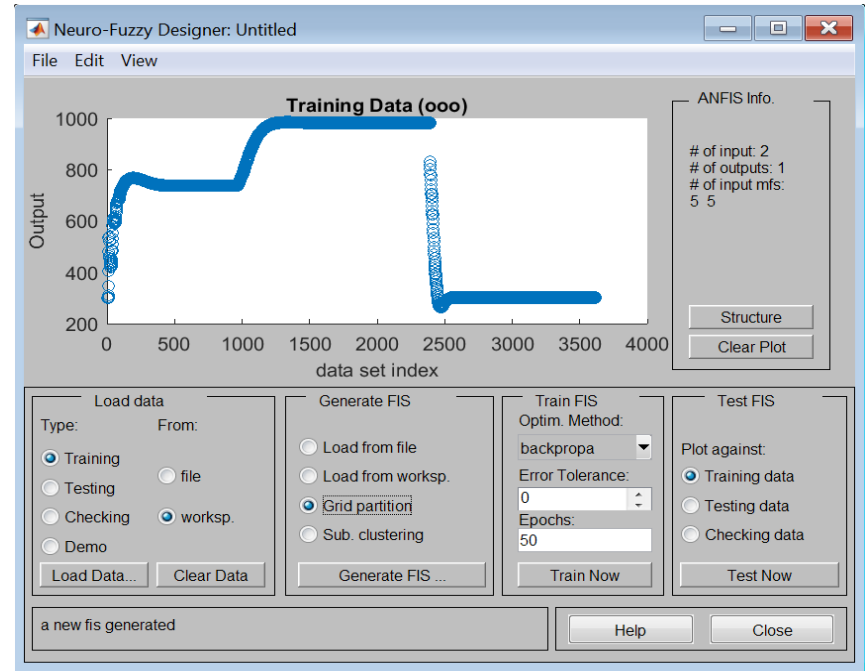

Figure2- ANFIS Editor

To start the GUI, type the following command at the MATLAB prompt:

anfisedit

The Neuro-Fuzzy Designer window shown in the following figure includes four distinct areas to support a typical workflow. The designer perform the following tasks:

1. Loading, Plotting, and Clearing the Data 
2. Generating or Loading the Initial FIS Structure

3. Training the FIS

4. Validating the Trained FIS

\subsection{Loading}

\section{Simulation Results}

To train an FIS, you must begin by loading a Training data set that contains the desired input/output data of the system to be modeled. Any data set must be an array with the data arranged as column vectors, and the output data in the last column. The training data set is prepared in this work using PID controller based conventional controller model. The input data set is speed error and rate of change of speed error and output data set is PID controller regulated output.

Training Data Set

\begin{tabular}{|l|l|l|}
\hline Speed error & Rate of change of speed & Output of controller \\
\hline 2000 & 0 & 300 \\
\hline 1999.99999999986 & -0.105058719514921 & 300.000200950893 \\
\hline 1999.99999999890 & -0.441642604165574 & 300.000526996623 \\
\hline 1999.99999999584 & -0.956899023726873 & 300.001007204438 \\
\hline 1999.99999995105 & -2.79789802870158 & 300.003408239095 \\
\hline 1999.99999899040 & -12.0029782817224 & 300.015413301865 \\
\hline 1999.99997576848 & -58.0296153549624 & 300.075435852631 \\
\hline 1999.99939913174 & -288.193317966132 & 300.375479491597 \\
\hline 1999.98499517190 & -1439.77125888300 & 301.873965102891 \\
\hline 1999.62402749242 & -7216.22242799417 & 309.322491672935 \\
\hline 1990.49187560698 & -36512.7644366602 & 345.395467626772 \\
\hline 1940.90940484708 & -107686.732070847 & 405.962336437442 \\
\hline 1778.07653715236 & -228719.675378755 & 481.297034179249 \\
\hline 1425.77754674369 & -400140.363727090 & 535.178362868541 \\
\hline 1033.61946227049 & -577535.744053745 & 539.430494659332 \\
\hline 1033.61946227047 & -655360 & 539.430494659331 \\
\hline 691.147375238024 & -679394.607450491 & 520.735451285145 \\
\hline 691.147375238005 & -704512 & 520.735451285143 \\
\hline 691.147375237985 & -696320 & 520.735451285142 \\
\hline 117.387798757541 & -605966.193377361 & 462.030132667526 \\
\hline 66.6948967038663 & -437557.514865671 & 455.222874366140 \\
\hline 29.8917482962902 & -404710.596662998 & 450.030269803560 \\
\hline 29.8917482962686 & -389120 & 450.030269803557 \\
\hline & &
\end{tabular}

To load a data set using the Load data portion of the designer:

a. Specify the data Type.

b. Select the data from a file or the MATLAB workspace.

c. Click Load Data.

After you load the data, it displays in the plot. The training, testing and checking data are annotated in blue as circles, diamonds, and pluses respectively.

To clear a specific data set from the designer:

a. In the Load data area, select the data Type.

b. Click Clear Data.

\subsection{Generating the Initial FIS Structure}

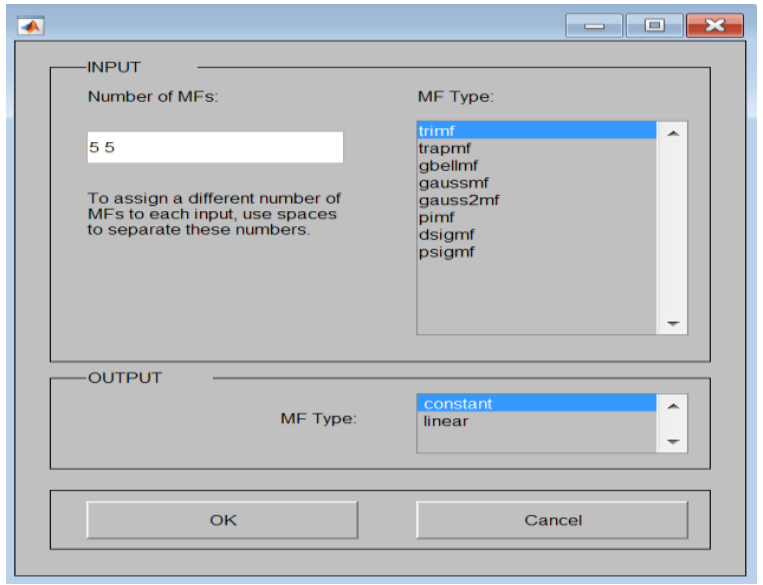

Figure3- FIS structure generating structure 
Before you start the FIS training, you must specify an initial FIS model structure. To specify the model structure, perform one of the following tasks:

Load a previously saved Sugeno-type FIS structure from a file or the MATLAB workspace. Generate the initial FIS model by choosing one of the following partitioning techniques:

Grid partition - Generates a single-output Sugeno-type FIS by using grid partitioning on the data.

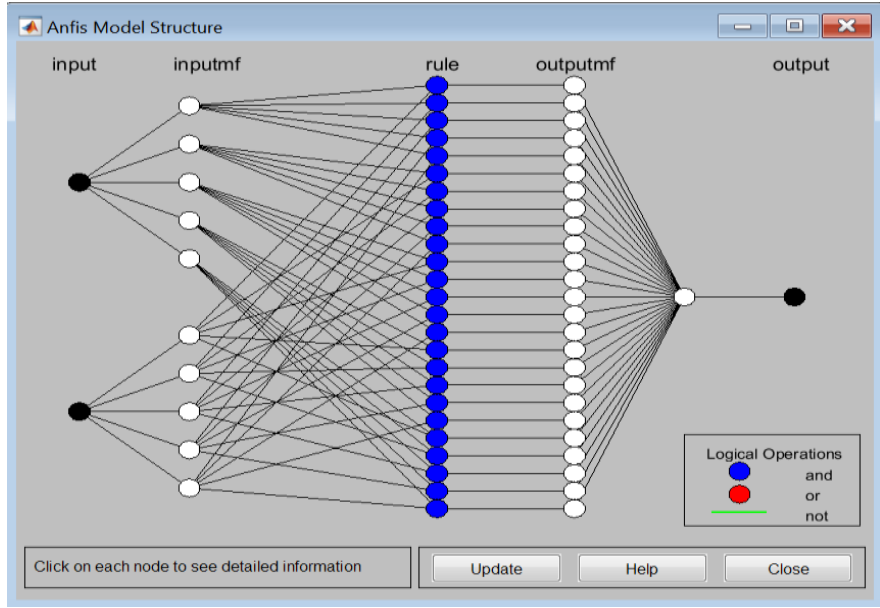

Figure4- FIS model structure

To view a graphical representation of the initial FIS model structure, click Structure.

\subsection{Training the FIS}

After loading the training data and generating the initial FIS structure, you can start training the FIS.

The following steps show you how to train the FIS.

1) In Optimum. Method, choose hybrid or back propagation as the optimization method.

The optimization methods train the membership function parameters to emulate the training data.

2) Enter the number of training Epochs and the training Error Tolerance to set the stopping criteria for training.

The training process stops whenever the maximum epoch number is reached or the training error goal is achieved.

3) Click Train Now to train the FIS.

This action adjusts the membership function parameters and displays the error plots.

After training, this information about ANFIS is generated in MATLAB Command prompt.

ANFIS info:

Number of nodes: 75

Number of linear parameters: 25

Number of nonlinear parameters: 30

Total number of parameters: 55

Number of training data pairs: 3614

Number of checking data pairs: 0

Number of fuzzy rules: 25

Start training ANFIS ...

1) 736.976

2) 736.969

Designated epoch number reached --> ANFIS training completed at epoch 2.Using a given input/output data set, the toolbox function anfis constructs a fuzzy inference system (FIS) whose membership function parameters are tuned (adjusted) using either a back propagation algorithm alone or in combination with a least squares type of method. After completion of training following sugeno fuzzy FIS file is generated. 


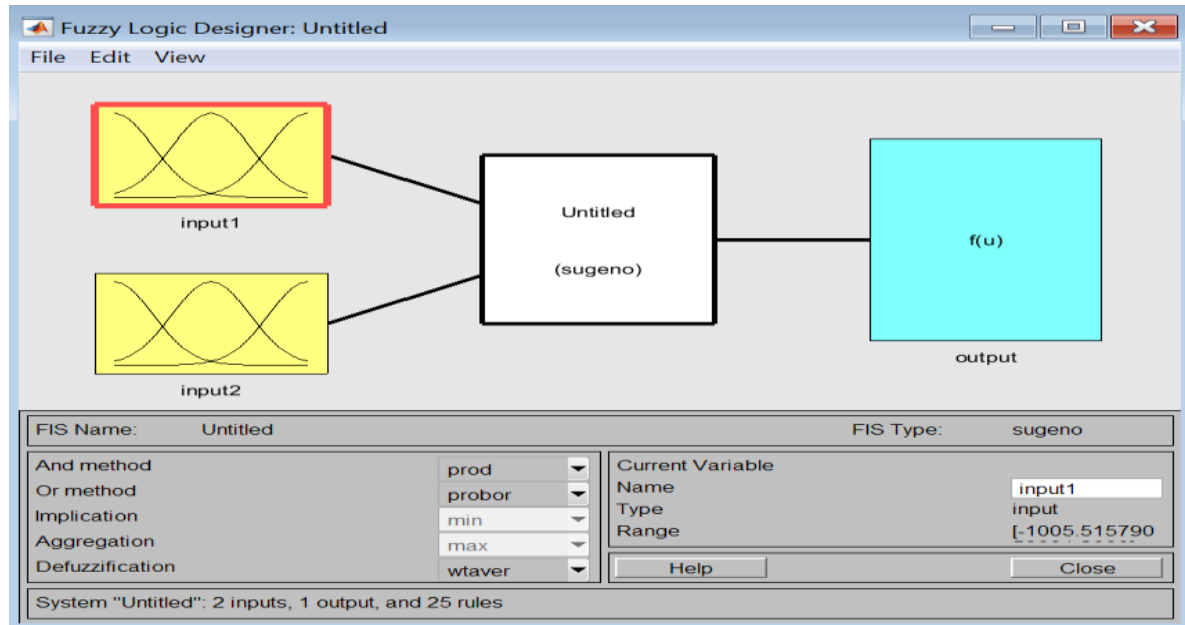

Figure5- Fuzzy logic generated using ANFIS

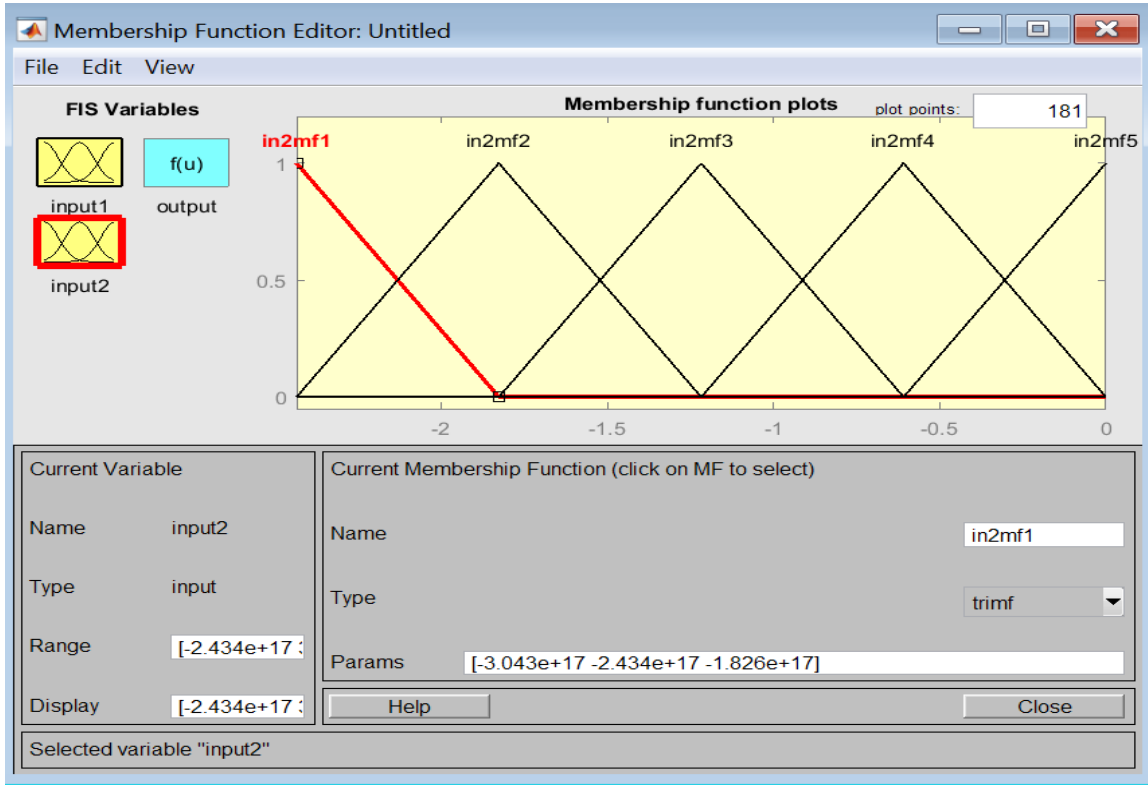

Figure6- Input2 Membership Function of Fuzzy logic using ANFIS

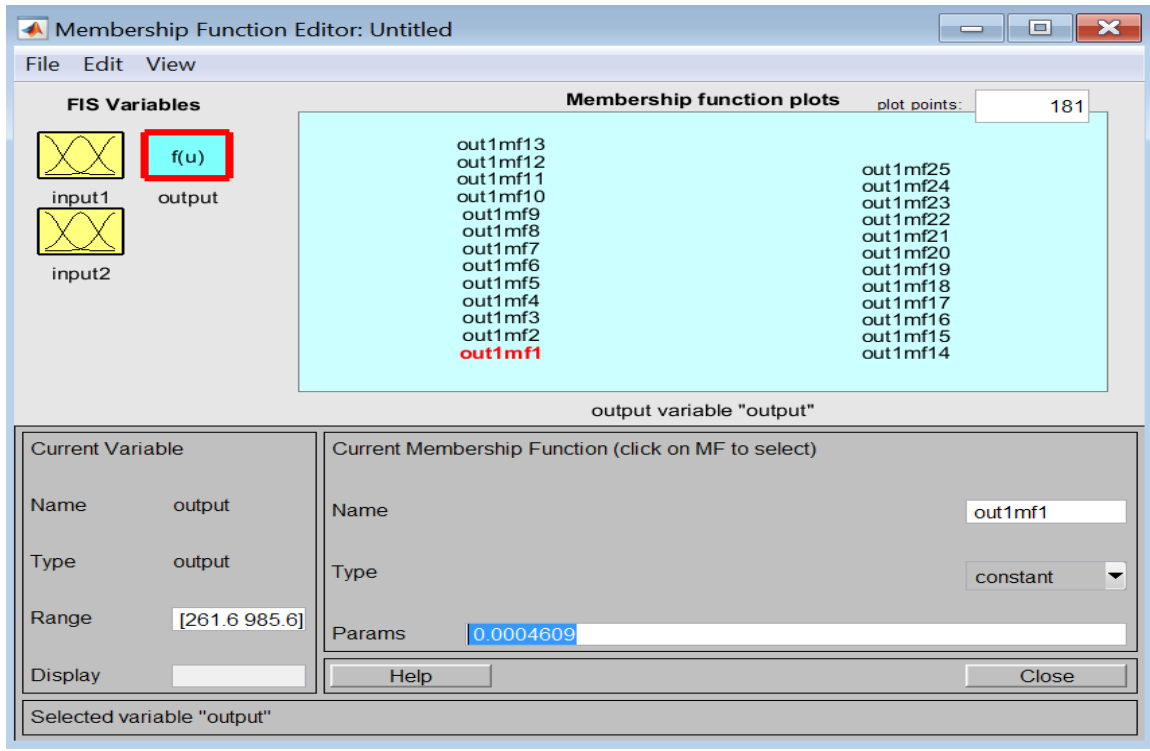

Figure7- Output Membership Function of Fuzzy logic using ANFIS 


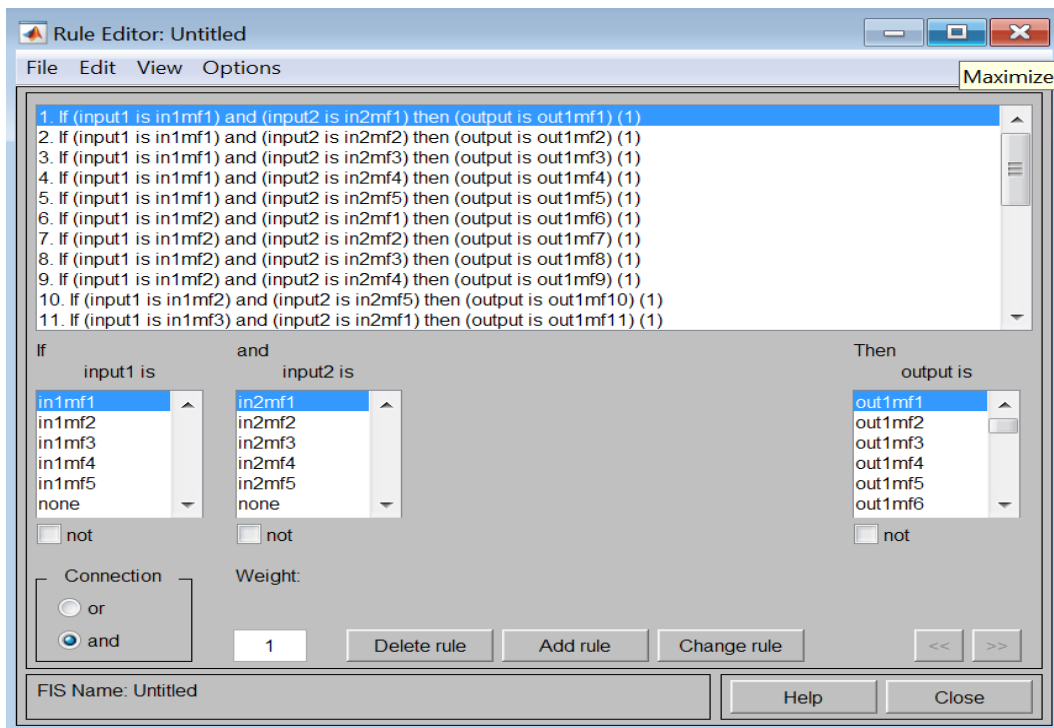

Figure8- Rules of Fuzzy logic using ANFIS

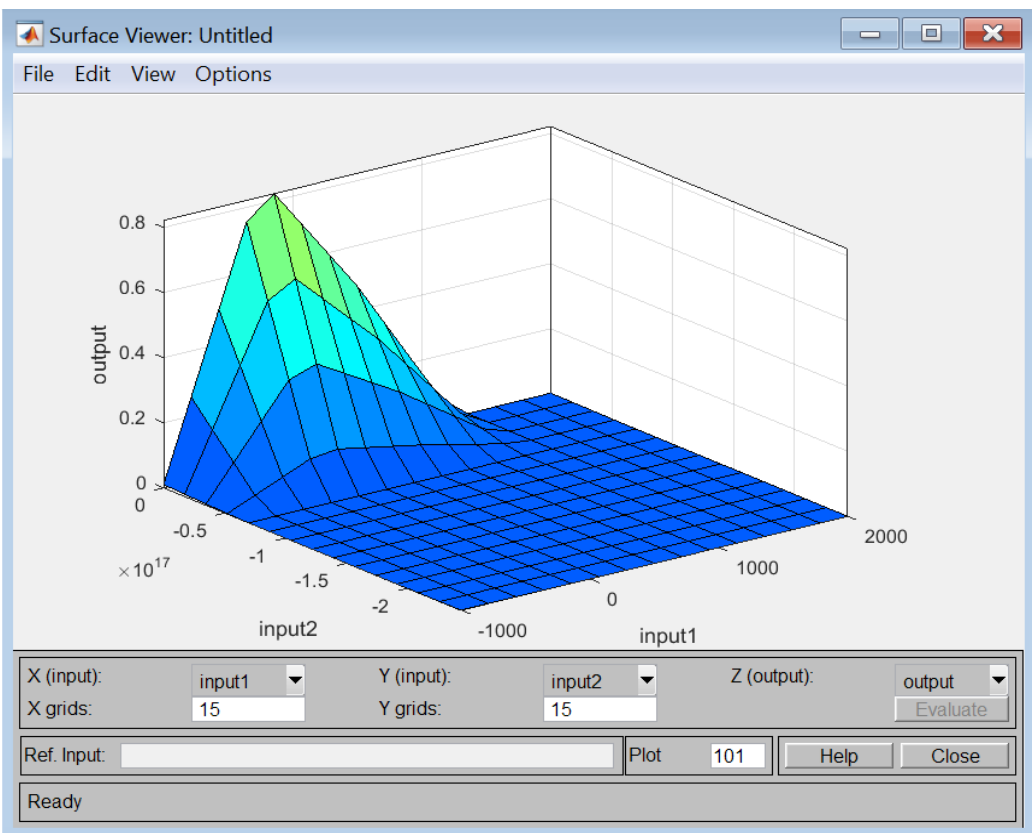

Figure9- Surface viewer of Fuzzy logic using ANFIS

1.4 Results

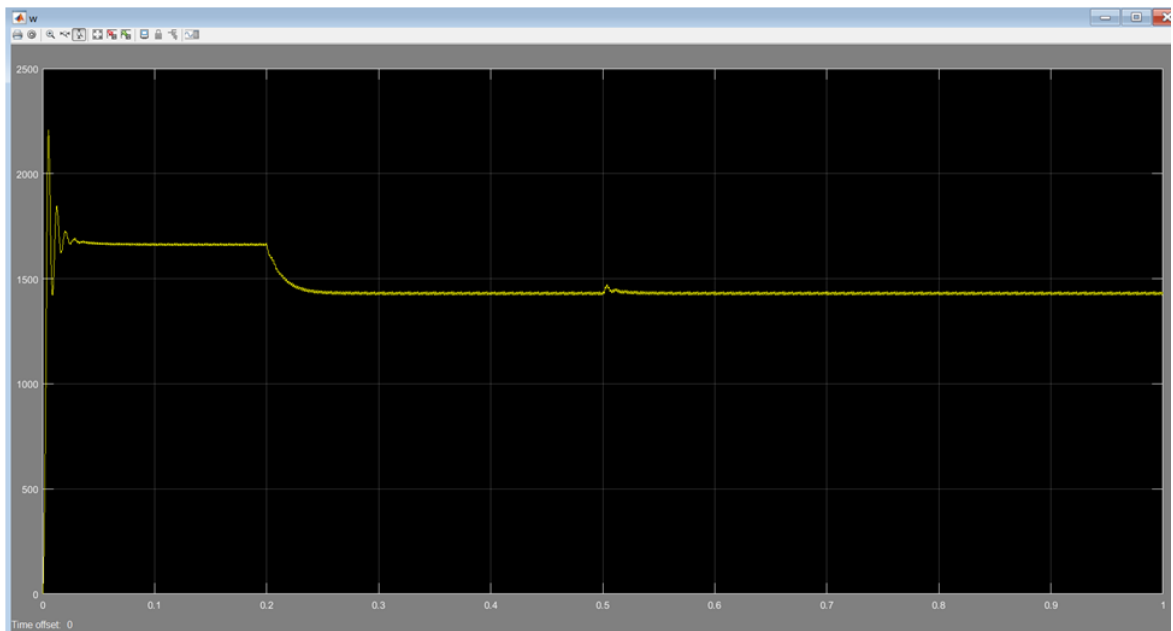

Figure 10 Regulated Speed of BLDC using ANFIS 
Regulated speed output of BLDC motor is according to Reference speed is 1660 which is change at $t=0.2 \mathrm{~s}$ to 1430 .

\section{Conclusion}

A logical method of acquiring the speed control of BLDC Motor drive by process of Adaptive NeuroFuzzy Inference System (ANFIS) has been explored in this research paper. The modeling and the simulation of the motor drive is explained in this paper. The exercise of training of membership functions is done in ANFIS, which is not taken charge of in the fuzzy counterpart. The outstanding gain of ANFIS is that it increase the dynamic performance and to provide good stabilization. It is additionally providing efficient and best results while working with linear techniques, optimization and adaptive techniques. It is experimentally verified by using MATLAB/SIMULINK software kit.

\section{References}

[1]. Research Inventy: International Journal Of Engineering And Science Vol.2, Issue 11 (April 2013), Pp 06-13 Issn(e): 2278-4721, Issn(p):2319-6483

[2]. Yong Liu, Z. Q. Zhu, and David Howe, "Commutation -Torque -Ripple minimization in direct -torque-controlled PM brushless DC drives," IEEE Trans. On industry applications, vol. 43, no.4, July/August 2007.

[3]. Neethu U. and Jisha V.R. , "Speed control of brushless de motor: A comparative study" 2012 IEEE International conference on power electronics, drives and energy systems, December 16-19, 2012, Bengaluru, India.

[4]. P.D.Evans and D.Brown, "Simulation of brushless DC drives", Proc. Of the IEEE, vol.137, no.5, pp. 299-308, September 1990

[5]. T.J.E. Miller, "Brushless permanent magnet and reluctance motor drive", Oxford 1989.

[6]. J.S. R. Jang, C.T. Sun and E. Mizutani, "Neuro-Fuzzy and Soft Computing", PTR Prentice Hall, (1997)

[7]. J.S.R. Jang, Adaptive Network Based Fuzzy Inference System”, IEEE Trans. On Systems Man. and Cybemetics, Vol.23, No.3, (1993), Pp 665-685.

[8]. Sakshi Solanki "Brushless DC motor drive during speed regulation with Artificial Neural Network Controller" Int. Journal of Engineering Research and Application ISSN : 2248-9622, Vol. 6, Issue 6, ( Part -3) June 2016, pp.01-05 